\title{
Quality assurance in planning a radon measurement survey using PDCA cycle approach: what improvements?
}

\author{
Giuseppe La Verde ${ }^{1,2, *}$, Vincenzo Roca ${ }^{1,2}$, and Mariagabriella Pugliese ${ }^{1,2}$ \\ ${ }^{1}$ Department of Physics "E. Pancini", University of Naples Federico II, Napoli, Italy \\ ${ }^{2}$ National Institute of Nuclear Physics, Section of Naples, Naples, Italy
}

Received: 14 November 2018 / Accepted: 16 March 2019

\begin{abstract}
This work is focused on effectiveness of adopting the Plan, Do, Check, Act (PDCA) cycle model, also known as Deming cycle, for an analytical laboratory management. It is not uncommon for university laboratories, unlike those of private companies, to register problems and losses in the management of materials, supplies and quality of service, especially externally. The PDCA cycle is closely associated with the planning, implementation, control and continuous improvement of the processes. In this context, the Deming cycle method finds a simple and informal application in order to make small modifications and improvements to the entire Radon gas activity concentrations measurement procedure. Attention is paid to most important topics as management of equipment and materials, record maintenance, sample analysis and risk communication. The results recorded were positive in terms of material savings, job optimization, quality of results and organization of internal processes.
\end{abstract}

Keywords: PDCA cycle / quality control / radon / LR115

\section{Introduction}

The importance of the role of the university concerns both the production of skills and knowledge to create qualified professionals and to be a bridge between the graduates and the world of work.

Nowadays, universities are asked to be closer to society's problem and cooperate with private companies to find solution or simply to offer a service. So, university testing laboratories become centers of reference for carrying out of particular measures for purposes not only scientific but also for legislative requirements.

The Laboratory of Radioactivity (LaRa) of the Department of Physics "E. Pancini" of the University of Naples Federico II is a testing laboratory for determination of concentration of specific activity of natural and artificial radionuclides in many matrices: air, soil, water and food products. The data are used for many purposes including environmental surveys and dose assessment in man and environmental pollution, the establishment of standards of regulatory guides, and decision-making on economic, legal and health aspects. So, scientific expertise and continuous research are available to private or public companies and therefore it was necessary to subject the management of the

\footnotetext{
* Corresponding author: glaverde@na.infn.it
}

laboratory to standardized protocols that could ensure the performance, control and monitoring of the operational activities.

Since 2011 the Laboratory of Radioactivity was certified ISO 9001. UNI EN ISO 9001 is a usual standard for quality management systems applicable to all organizations irrespective of kind, size or product or service provided. Therefore, it is also applicable to laboratories, even though its language is generic. Its purpose is to specify a quality management system that will allow an organization to demonstrate its ability to provide a product that meets customer and applicable regulatory requirements. It also aims to enhance customer satisfaction, including processes for continue improvements and assurance of conformity [1].

In 2013 the management of the Laboratory of Radioactivity decided to develop an internal quality system, to adopt the international standard ISO 17025:2005 and to obtain qualification for the measurement of concentration of radon gas activity in air, using passive LR115 detectors, according to recommendations of reference ISO $[2,3]$. The standard of ISO was validated by intercomparison at BundesamtfürStrahlenschutz (BfS). UNI CEI EN ISO/IEC 17025 was developed as a special purpose standard for laboratories to specify the general requirements for their technical competence. While the standard is generic, on the other hand it recognizes that for 
accreditation purposes (i.e. for recognition of competence to perform specific tests or calibrations) the standard may require development of guidelines to explain its use in specific areas of testing or measurement [4].

But the most important requirement for the success of a quality system is to perform a high-quality work through competent and dedicated employees and intellectual honesty in communicating the results obtained. Anyway, the leading principle is to perform corrective actions whenever substandard results are detected. It therefore becomes natural that an approach based on monitoring, correction and improvement (dictated by the protocols of standardized procedures) can become a philosophy of life within the laboratory.

The Plan, Do, Check, Act (PDCA) cycle model represents this evolution as it involves not only the correct execution of the laboratory activity but it also influences its approach, stimulating the critical and analytical sense of those who work. The Deming cycle scheme includes 4 basic steps [5]:

- PLAN: what to do, how to do it.

Establish the objectives and processes necessary to deliver results according to needs/expectations;

- DO: do what planned.

Putting the planned actions into practice according to established procedures and timelines;

- CHECK: check that what is planned is done.

Monitor and measure the processes and characteristics of the service/action, verifying that there is consistency between what is achieved and what has been planned;

- ACT: continuously improve.

Periodically check that the system is consistent with what is planned and take the appropriate actions for the continuous improvement of operational, organizational and management processes.

With this new organizational structure adopted by LaRa, a large-scale campaign of indoor radon gas concentration measurements was organized, in order to validate the method adopted. The measurements were conducted using passive detectors, each equipped with a pair of LR115 solid-state nuclear track detectors (SSNTD). The detectors were exposed in the rooms where the inhabitants spent the most of their time, generally the living room and bedroom, for 1 yr (this period was divided into two semesters: summer and winter, and so two measurements). The measures and improvements that have been made to the whole process, including the organization, the carrying out of the measures and the management of data and materials used, will be described below.

\section{Materials and methods: the quality system of LaRa}

LaRa, according to international standards, has developed an internal quality program according to the working context of the laboratory itself. The basic scheme is shown in Figure 1: where the required inputs and expected

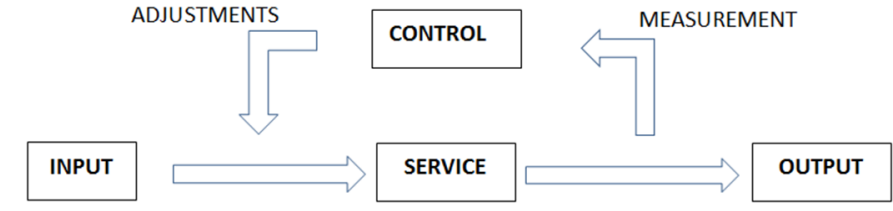

Fig. 1. Flowchart of an ISO 9001 general process.
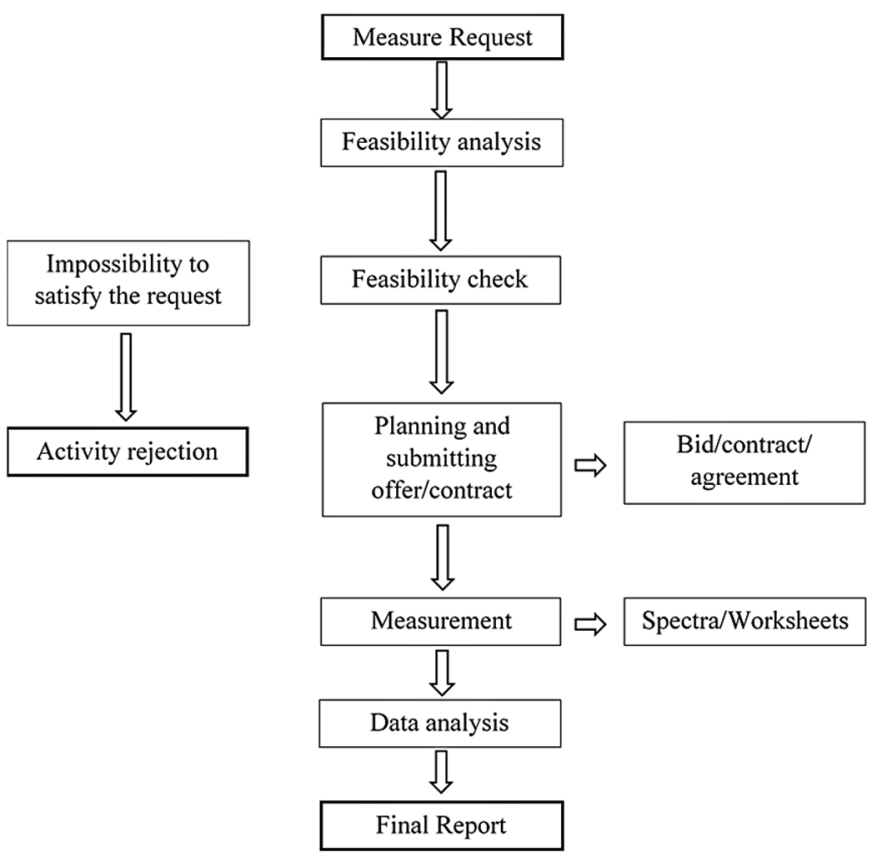

Fig. 2. Internal organization of LaRa in according to ISO 9001.

outputs for each process are considered. Inputs are generally received from another process and may be materials, information, documents, services, semi-finished products, etc. The outputs, on the other hand, consist of what comes out of a process at its end and can feed another process as input. The processes must be verified in some way to make sure that they get the results for which they were designed so we need to establish: criteria, methodologies, measurements and indicators to ensure effective operation and process control. This represents the control phase of the whole process through which it is possible to determine the potential improvement categories (adjustments) that are applicable to the processes and thus obtain an output product as close to the desired characteristics.

The organization of the ISO system in the laboratory follows a flow chart that traces its basic approach (Fig. 2).

For LaRa's internal quality program, the main objectives are:

- to have an updated calibration

- to control process;

- to check the reliability of the results.

In order to achieve accreditation all assays must provide:

- method validation;

- correct development and register of operations; 
- equipment calibration;

- internal control activities (measurements of blanks, replicates and reference materials as well as retesting);

- external control activities (intercomparison and proficiency tests);

- calculation of uncertainty;

- expression of results.

All tests are structured in six steps, in order to make documentation easy to consult and to manage the traceability of process:

1. acceptance and registration of sample;

2. pre-treatment;

3. treatment;

4. measurement;

5. data analysis;

6. final report.

For external quality control, LaRa takes part in intercomparison for evaluation of its analytical performance above all for measurements of activity concentration of radon gas in air. For these kind of measurements passive detectors such as E-PERM electret detector and SSNTD (LR115) are used. In particular, in the case of LR115 detectors, a method for Track counting and thickness measurement using a commercial image scanner was developed [6,7]. LaRa has been certified ISO 17025:2005 by Accredia, the national accreditation authority, in 2014. Since then, the interlaboratory participation schedule is established and approved annually and it took place at BfS Radon Calibration Service Laboratory (Germany). BfS is accredited by the German Accreditation Body Deutsche Akkreditierungsstelle $\mathrm{GmbH}$ (DAkkS) according to the most recent norm EN ISO/IEC17025:2017 for the measurands radon-222 activity concentration and potential alpha energy concentration of short-lived radon-222 decay products.

The equipment of the Radon Calibration Service Laboratory consists of two walk-in chambers $\left(30 \mathrm{~m}^{3}\right.$ and $11 \mathrm{~m}^{3}$ ) and five $0.4-\mathrm{m}^{3}$-containers all made of stainless steel, and of supplementary technical devices. Each unit, i.e. the chambers and the five containers, is equipped with sensors to measure quantities of radon activity concentration, temperature and humidity. For the purpose of measuring radon activity concentration flow-through scintillation cells, traced back to national standard, are used. With this equipment all parameters influencing the reference atmosphere are monitored permanently. The values from these on-line measurements are displayed on the screen of the control panel and are continuously registered. The radon activity concentration in the containers can be adjusted to values in the range from 500 to $100000 \mathrm{~Bq} \mathrm{~m}^{3}$.

Results obtained from all intercomparisons have been conformed to the required parameters. For LaRa the results are considered positive if the relative variation of the experimentally determined exposure is $\leq 20 \%$.

Internal and external quality control procedures compose the total quality management (TQM) system.

To involve and stimulate staff, it was decided that all members should follow one or more annual update of the quality system in order to ensure its sharing and verify its effectiveness.
Furthermore, the quality manual is accessible to everyone, including external collaborators, and has a simple structure that is easy and immediate to understand.

\subsection{Error and uncertainty of results}

The result of a measurement, even in the absence of known systematic errors, is only an estimate of the true value of the measurand due to the uncertainty originated by the random effects and by the systematic effects not known or not considered. The result of a measurement is expressed correctly and completely only if it also includes the expression of the uncertainty associated with the measurand. The uncertainty associated with the result of a measurement provides information on the dispersion of values reasonably attributable to the result.

For integrated measurement method to determine average activity concentration using LR115 detectors, in according to ISO 11665-4:2015's directions, the average concentration of radon activity $\bar{C}$ is obtained by the following formula:

$$
\bar{C}=\frac{\left(N_{g}-\bar{N}_{b}\right)}{[1+\beta(s-6.5)] \cdot S_{S S N T} \cdot t \cdot F_{C}}
$$
by

And the standard uncertainty composed of $\mathrm{C}$ is given

$$
u(\bar{C})=\frac{1}{F_{c} t} \sqrt{u^{2}\left(R_{s}\right)+R_{s}^{2} \cdot u_{r e l}^{2} \cdot\left(F_{c}\right)}
$$

where

$$
\begin{aligned}
& u\left(R_{s}\right)=\frac{1}{\lfloor 1+\beta \times(s-6.5)\rfloor} \\
& \sqrt{\left(\frac{N_{g}-\overline{N_{b}} / n}{\left(S_{S S N T D}^{2}\right)}\right)+\left[R_{s}^{2} \times\left[(s-6.5)^{2} \times u^{2}(\beta)+u^{2}(s) \times \beta^{2}\right]\right]}
\end{aligned}
$$

$N_{\mathrm{g}}$ is the number of tracks after exposure, $N_{b}$ the average number of tracks caused by background, $\beta$ the correction coefficient for thickness (number of tracks $/ \mu \mathrm{m}$ ), $s$ the residual thickness of the detector $(\mu \mathrm{m}), S_{S S N T}$ the nuclear solid-state detector area used for trace counting $\left(\mathrm{cm}^{2}\right), t$ the sampling time $(\mathrm{h}), F_{c}$ the calibration factor (number of tracks $\left./ \mathrm{cm}^{2}\right) /\left(\mathrm{Bqh} / \mathrm{m}^{3}\right), u$ the uncertainty associated with the measurement result, $R_{s}$ thickness, $u_{\text {rel }}$ the relative standard uncertainty and $n$ the number of detectors used to determine the background.

The extended uncertainty of $\bar{C}$ is given by: $U=k u(\bar{C})$ with $k=2$. 


\section{The case of radon activity concentration measurements in air}

In 2014 LaRa carried out a campaign for measuring the activity concentration of radon gas in dwellings in Puglia, South Italy [8]. The purpose was to make a preliminary mapping of the distribution of gas activity concentrations in domestic environments.

An improved protocol was planned matching to the normal operating protocol, as in Figure 3a, and following the principles of the PDCA cycle. It took into account aspects never before considered: first above all in-depth information on the methods of conducting the study and correct risk communication to the population concerned, as reported in Figure 3b below:

In support of this new aspect, informative brochures were designed and presentation seminars were organized that contributed to make citizens aware.

For internal organization of the management of laboratory resources, particular importance has been given to the reduction of material waste and process optimization. Main changes have been performed:

1. to assign a delivery receipt to each detector in which to record personal data;

2. to plan days to start the measures synchronously;

3. use of molds to cut out the aluminized paper that serves as a protective envelope for the detectors;

4. plan the chemical development of the films in such a way as to optimize the quantity of reagent and number of films (possible if and only if point 2 has occurred).

\section{Results and discussion}

In all wide measurement campaigns, one of the big problems is the complete pick up of the detectors. That occurs because the detectors reach different kinds of users that, not having received useful and complete information on the purpose of the measurement, often ignore its importance. For this reason process improvements have been designed to implement the dissemination of information about the survey, the radon risk and the importance of using the detectors.

In studies carried out in previous years [9-13], there has been a significant loss of detectors. In the publications of works, this data has not been reported; however, the outgoing and picked-up detectors have been recorded in an internal register. As shown in Figure 4, there was a loss of detectors of $19 \%$. One detector is considered lost when it has not been found or if it has been found but their reading is not possible.

This implied strong outcomes on both economic aspect and data analysis (Fig. 4).

In the radon measurements survey of 2014, the dissemination played an important role. Following the communication model shown in Figure 5 and also mentioned by the Istituto Superiore di Sanità through a dedicated channel, called Piano Nazionale Radon [14] (Fig. 5).

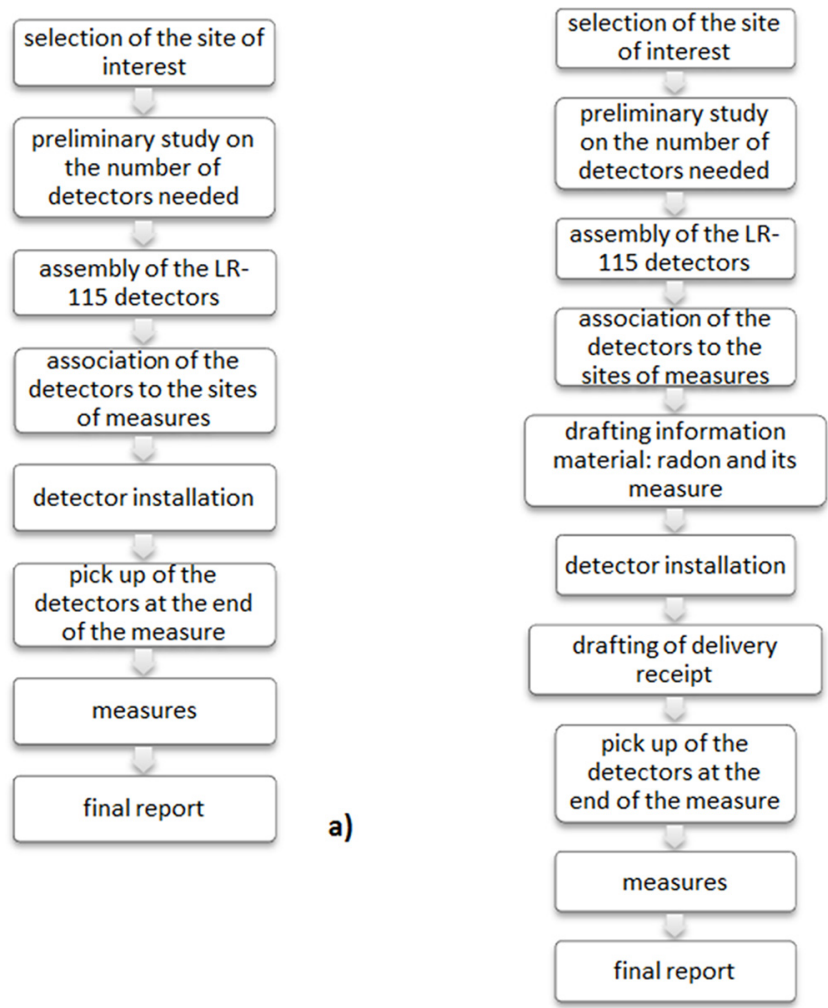

b)

Fig. 3. (a) Standard flowchart of LaRa; (b) new flowchart for measurement's campaign in Puglia.

pick up of detectors

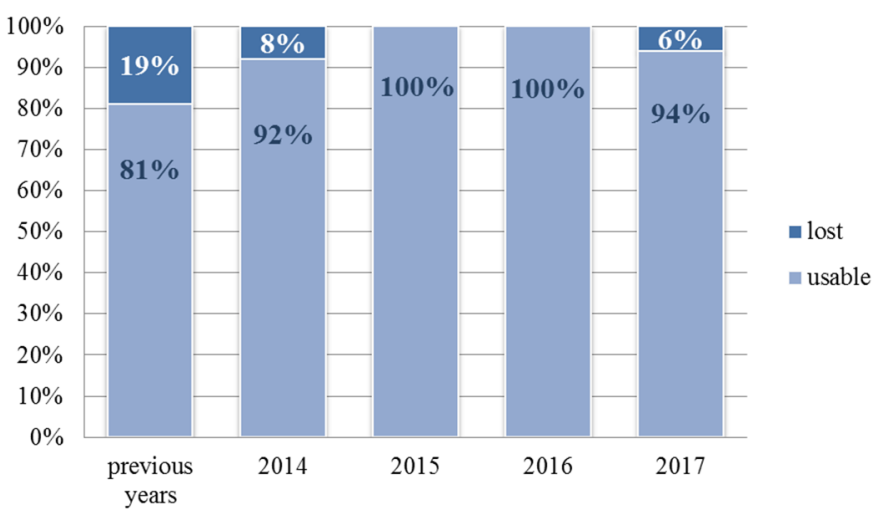

Fig. 4. Percentage pick-up rate of the detectors.

The success of an information campaign is related to the concept of risk perception, so, once resources have been selected (including qualified personnel), information must be balanced (without the risk to create alarmism or to underestimate the problem) and usable by the greatest number of people [15]. Radon exposure in indoor environments increases the risk of contracting lung cancer and, for some countries, it is estimated to be responsible for a percentage ranging from $3 \%$ to $14 \%$ of all lung cancers [16]. Specifically, the inhaled decay products, mostly attached 


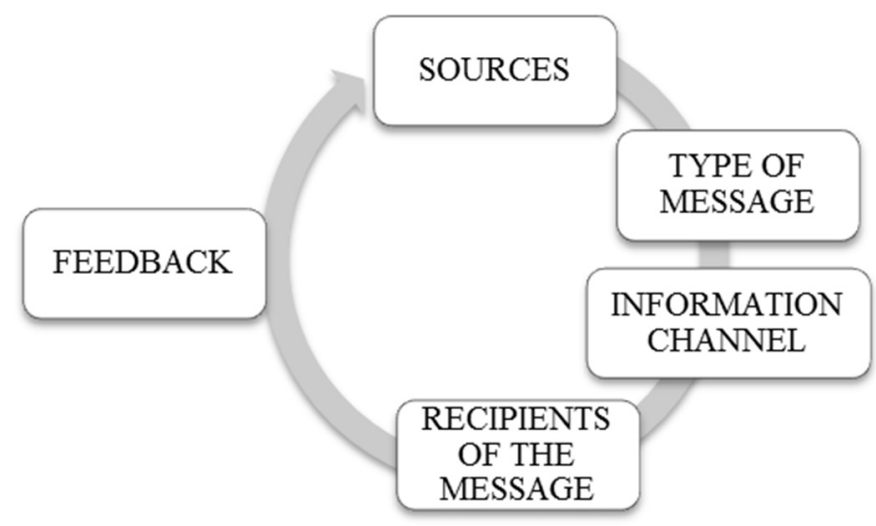

Fig. 5. Standard organization model for dissemination used by ISS.

to the particulate always present in the air, are deposited on the walls of the respiratory system and from here they irradiate (above all through the alpha radiations) the cells of the bronchi. Radiation in some cases produces damage to the DNA of such cells damage that, if not properly repaired by the appropriate cellular mechanisms, can evolve into a lung cancer. For this reason the World Health Organization (WHO), through the International Agency for Research on Cancer (IARC), has classified radon in Group 1 , which lists the substances for which there is a sufficient evidence of carcinogenicity based on epidemiological studies in humans [17].

In addition, providing a delivery receipt of the detectors to the people informed on the subject, it increases the sense of responsibility and of involvement throughout the measurement process. At the same time, as shown in Figure 4, a reduction from $19 \%$ to $8 \%$ of lost detectors has been occurred and this reduction was also recorded for the measurement campaigns carried out in the following years.

Another improvement in the sample management process was the synchronization of the start of the measurements. The adoption of continuous improvements is not always easy for laboratory operations, but in this way the pick-up of detector and film LR115 development have been optimized. It was possible to use the maximum number of films allowed for a single chemical treatment saving about $37.5 \%$ of $\mathrm{NaOH}$. As reported in Figure 6, if about $12 \mathrm{~L}$ of $\mathrm{NaOH}$ were usually needed to develop 100 films, for this survey 7.5 L solution are enough for the same number of films (Fig. 6).

With the same optimization approach, the process of preparing protective packaging in aluminized paper was managed. By preparing some shapes to cut out, around $8 \%$ of the waste was saved.

\section{Conclusions}

An approach based on continuous improvement can produce benefits to quality process, according to ISO 9001:2015, and to reliability of analytical data, as prescribed by the ISO 17025:2017.

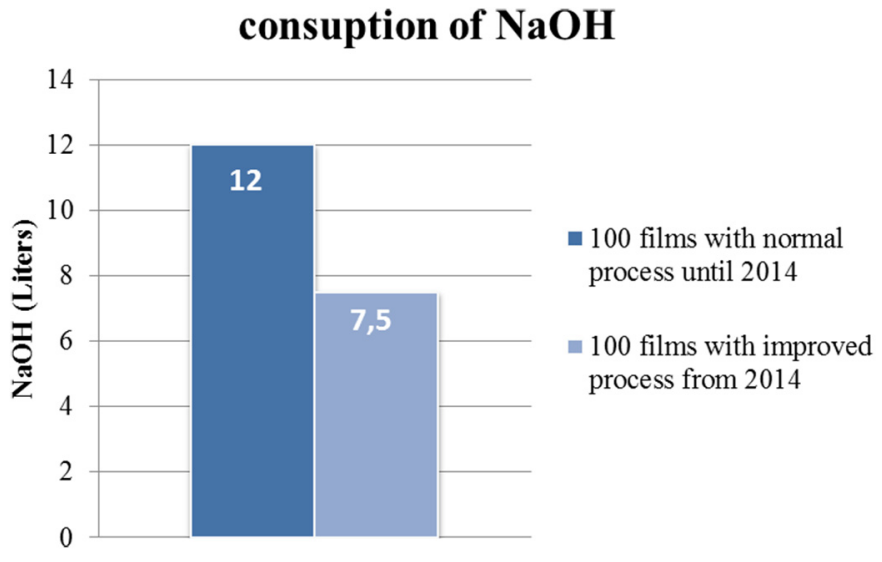

Fig. 6. Average consumption of $\mathrm{NaOH}$ used for the development of 100 LR115 films.

The main results can be summarized as follows:

1. higher recovery rate of detectors at the end of measurement;

2. waste reduction of materials and reagents;

3. increase of correct information and knowledge on the subject of radon gas and its daughters inhalation.

The positive feedback found in the experience of this work represents an important step, whose standardization is under way and represents the basis on which to plan a further improvement and PDCA cycle adopted by the Laboratory of Radioactivity becomes its source of inspiration.

\section{References}

1. UNI EN ISO 9001:2015. Quality management systems Requirements

2. ISO 11665-1:2015. Measurement of radioactivity in the environment - Air: radon-222-Part 1: Origins of radon and its short-lived decay products and associated measurement methods

3. ISO 11665-4:2015. Measurement of radioactivity in the environment - Air: radon-222-Part 4: Integrated measurement method for determining average activity concentration using passive sampling and delayed analysis

4. UNI CEI EN ISO/IEC 17025:2017. General requirements for the competence of testing and calibration laboratories

5. K. Ishikawa, Guida al controllo della qualità (FrancoAngeli, Milano, 1988)

6. F. De Cicco, M. Pugliese, V. Roca, C. Sabbarese, Dependence of the LR-115 radon detector calibration factor on track density, Appl. Radiat. Isot. 78, 108-112 (2013)

7. F. De Cicco, M. Pugliese, V. Roca, C. Sabbarese, Track counting and thickness measurement of LR115 radon detectors using a commercial image scanner, Radiat. Prot. Dosimetry. 162, 388-393 (2014)

8. M. Quarto, M. Pugliese, G. La Verde, F. Loffredo, V. Roca, Radon exposure assessment and relative effective dose estimation to inhabitants of Puglia Region, South Italy, Int. J. Environ. Res. Public Health 12, 14948-14957 (2015)

9. M. Pugliese, V. Roca, G. Gialanella, 222Rn indoor concentration in Campania, Phys. Med. 10, 118-119 (1994) 
10. F. Bochicchio, G. Campos-Venuti, S. Piermattei, C. Nuccetelli, S. Risica, L. Tommasino, G. Torri, M. Magnoni, G. Agnesod, G. Sgorbati, M. Bonomi, L. Minach, F. Trotti, M.R. Malisan, S. Maggiolo, L. Gaidolfi, C. Giannardi, A. Rongoni, M. Lombardi, G. Cherubini, S. D'Ostilio, C. Cristofaro, M. Pugliese, V. Martucci, A. Crispino, P. Cuzzocrea, A.S. Santamaria, M. Cappai, Annual average and seasonal variations of residential radon concentration for all the Italian Regions, Radiat. Meas. 40, 686-694 (2005)

11. G. Venoso, F. De Cicco, B. Flores, L. Gialanella, M. Pugliese, V. Roca, C. Sabbarese, Radon concentrations in schools of the Neapolitan area, Radiat. Meas. 44, 127-130 (2009)

12. M. Quarto, M. Pugliese, F. Loffredo, V. Roca, Indoor radon concentration measurements in some dwellings of the Penisola Sorrentina, South Italy, Radiat. Prot. Dosimetry 156, 207-212 (2013)
13. M. Quarto, M. Pugliese, F. Loffredo, V. Roca, Indoor radon concentration and gamma dose rate in dwellings of the Province of Naples, South Italy, and estimation of the effective dose to the inhabitants, Radioprotection 51, 31-36 (2016)

14. Istituto Superiore di Sanità, Piano Nazionale, Radon, 2002. http://old.iss.it/binary/radon/cont/PNRtesto.pdf

15. R.E. Lundgren, A.H. McMakin, Risk communication: A handbook for communicating environmental, safety, and health risks, 6 edn. (John Wiley \& Sons, NJ, 2018)

16. World Health Organization, in: H. Zeeb, F. Shannoun (Eds.), WHO handbook on indoor radon: a public health perspective (WHO, Geneva, 2005)

17. IARC, IARC Monographs on the Evaluation of Carcinogenic Risks to Humans, Man-made Mineral Fibres and Radon (IARC, Lyon, 1988), Vol. 43

Cite this article as: Giuseppe La Verde, Vincenzo Roca, Mariagabriella Pugliese, Quality assurance in planning a radon measurement survey using PDCA cycle approach: what improvements? Int. J. Metrol. Qual. Eng. 10, 2 (2019) 